\title{
Heave motion suppression of a Spar with a heave
}

\section{plate}

\author{
Longbin $\mathrm{Tao}^{1 *}$ and Shunqing $\mathrm{Cai}^{2}$ \\ ${ }^{a}$ School of Engineering, Griffith University, PMB50 Gold Coast Mail Centre, Qld 9726, \\ Australia \\ ${ }^{\mathrm{b}}$ School of Civil and Resource Engineering, The University of Western Australia, 35 \\ Stirling Hwy, Crawley, WA6009, Australia
}

\begin{abstract}
Vortex shedding flow of an oscillating vertical cylinder with a disk attached at its keel is considered. This configuration is of interest for the offshore oil and gas industry. A finite difference method is employed to solve the incompressible Navier-Stokes equations in the primitive variables formulation. Test cases were used to guide selection of the size of flow domain, numerical parameters and verify that the resultant method was both convergent and accurate. Numerical simulations have shown that the geometry configurations of the cylinder and disk, such as aspect ratio of the disk $t_{d} / D_{d}$ and diameter ratio, $D_{d} / D_{c}$ have significant influence on the vortex shedding modes and associated hydrodynamic properties, e.g. hydrodynamic damping and added mass coefficients. Which in turn affect the performance in heave motion control of the structures.
\end{abstract}

Key words: finite difference method, vortex shedding, heave damping

\footnotetext{
* correspondence to Longbin Tao, Fax: $61-7-55528065$, E-mail: L.Tao@mailbox.gu.edu.au
} 


\section{Introduction}

Newly developed floating structure systems for offshore oil and gas industry operating in deep water, e.g. Tension Leg Platform (TLP) and Spar platform, have distinct advantage in relatively small magnitude of motion in vertical planes. This resulted in the wide application of the rigid risers. However, resonant heave oscillations may still occur due to nonlinear wave effects (sum-frequency) on TLPs and linear wave excitations of wave spectrum with long wave periods on Spars. These heave resonances are often excessive to cause damage in both risers and mooring systems.

In order to enhance the damping mechanism during the resonant oscillation of a TLP or Spar in the vertical direction, additional mechanical damping devices or other active damping systems are introduced externally. A typical example of these damping devices is heave plate which is attached to the keel of a TLP column or Spar (Fig. 1). The additional disk at the bottom of the column enhances the vortex shedding process, consequently changes the hydrodynamic properties by introducing extra damping and increasing the added mass of the system.

The two non-dimensional characteristic parameters representing the amplitude and frequency of the oscillation for cylinder and disk configuration are the KeuleganCarpenter number $(K C)$ and the frequency parameter $(\beta)$. They are defined as:

$$
\begin{aligned}
& K C=\frac{2 \pi a}{D_{d}} \\
& \beta=\frac{\left(D_{d}\right)^{2} f}{\nu}
\end{aligned}
$$

where $D_{d}$ is the diameter of the disk, $a$ and $f$ are the amplitude and frequency of the oscillation respectively and $\nu$ is the kinematic viscosity of the fluid. One can 
obtain a Reynolds number based on the flow oscillation velocity as:

$$
R_{e}=(K C) \beta=\frac{a \omega\left(D_{d}\right)}{\nu}
$$

Thiagarajan \& Troesch (1998) observed the flow of cylinder+disk configuration using the Particle Image Velocimetry (PIV) technique. The vortex shedding pattern was found to be dependent on both flow parameters $(K C, \beta)$ and the geometry of the structure. For a disk with two edges oscillating at small amplitude, the flow was found to be symmetric about the mean position of oscillation. Vorticity shed from the edges rolls up into vortex rings, which do not convect away from the disk. They remain in the proximity of the disk due to low $K C$, until flow reversal causes a rapid cancellation of vorticity. The measurements showed that the disk was found to increase the pressure drag coefficients $\left(C_{d}\right)$ two-fold. In the case that the thickness of the disk is very small, the two edges of the disk become virtually one single sharp edge, a well-established and reasonably stable vortex shedding pattern was generated by the single sharp edge. Vortex shedding occurred at a large angle of either positive or negative direction depending on starting condition. Thiagarajan (1993) noticed a difference in strengths of two consecutive vortices forming a pair, and interpreted that as due to a longer time period available for one of the vortices to develop depending on the starting direction of the oscillation.

Investigation of Tao (2002) showed that the three distinct vortex shedding modes, namely independent, interactive and uni-directional vortex shedding, occur due to different $K C$ and the thickness of the disk. Diffusion or convection was found to be predominant in each of the different vortex shedding regimes respectively. Consequently distinct hydrodynamic heave damping behaviours were found associated with different vortex shedding regimes, i.e. a lower, weakly $K C$-dependent damping was found in the independent vortex shedding regime, while the damping was 
found to be nonlinearly dependent on $K C$ in the later two vortex shedding regimes resulted from strong convection effect.

This paper is to conduct an investigation of a systematic variation of disk geometry, especially the influence of the diameter ratio, $D_{d} / D_{c}$, on the vortex shedding pattern and associated hydrodynamic heave damping arising from the flow separation and vortex shedding around the cylinder+disk. A finite difference solution of the viscous fluid structure interaction problem is obtained by solving NavierStokes equations for incompressible viscous flow. The present numerical method is validated against several benchmark problems and available experimental results. Recommendations on the design of heave plate for floating offshore structures are made based on the numerical calculation.

\section{Theoretical formulation}

\subsection{Governing equations}

The flow induced by a vertical cylinder with a disk attached at its keel oscillating at low $K C$ along its axis can be idealized as axi-symmetric by neglecting some threedimensional effects (see Fig. 1). The governing equations for the time dependent unsteady flow are the continuity and Navier-Stokes equations for incompressible viscous fluid. The axisymmetric coordinate system shown in Fig. 1 is fixed to the bottom open boundary with positive axes pointing right and upwards. The nondimensional form of the governing equations in cylindrical polar coordinate system $(r, y)$ are written as:

$$
\frac{1}{r} \frac{\partial(r u)}{\partial r}+\frac{\partial v}{\partial y}=0
$$




$$
\begin{aligned}
& \frac{\partial u}{\partial t}+(\mathbf{V} \cdot \nabla) u=-\frac{\partial p}{\partial r}+\frac{1}{R_{e}^{\prime}}\left(\nabla^{2} u-\frac{u}{r^{2}}\right) \\
& \frac{\partial v}{\partial t}+(\mathbf{V} \cdot \nabla) v=-\frac{\partial p}{\partial y}+\frac{1}{R_{e}^{\prime}}\left(\nabla^{2} v\right)
\end{aligned}
$$

here $\mathbf{V}=(u, v)$ is the velocity vector, $(u, v)$ denote radial and axial components of velocity respectively, $t$ time, and $p$ the dynamic pressure. In this paper, the governing equations and all physical quantities are presented in a non-dimensional form. $R_{e}^{\prime}=(R \sqrt{R g}) / \nu$ in Eqs. (5) and (6) arises due to the fundamental variables used in non-dimensionalization, i.e. density of fluid $\varrho$, acceleration of gravity $g$, and radius of the cylinder $R . \nabla$ and $\nabla^{2}$ are the gradient operator and Laplacian operator respectively.

The Poisson equation for pressure can be derived by taking divergence of the momentum Eqs. (5) and (6),

$$
\nabla^{2} p=-\frac{\partial \tilde{D}}{\partial t}-\left[\left(\frac{\partial u}{\partial r}\right)^{2}+\left(\frac{\partial v}{\partial y}\right)^{2}+2 \frac{\partial v}{\partial r} \frac{\partial u}{\partial y}\right]-\frac{u^{2}}{r^{2}}
$$

where $\tilde{D}$ is divergence, for axisymmetric flow

$$
\tilde{D} \equiv \nabla \cdot \mathbf{V} \equiv \frac{1}{r} \frac{\partial(r u)}{\partial r}+\frac{\partial v}{\partial y}
$$

\subsection{Boundary conditions}

Since the cylinder with a disk is forced to oscillate sinusoidally along its longitudinal axis (see Fig. 2) as

$$
y(t)=y(0)+a \sin (\omega t+\theta) \text { for } t>0,
$$


where $a, \omega$ are the amplitude and circular frequency of the oscillation respectively. No-flux and no-slip velocity boundary conditions are imposed along the oscillatory cylinder and disk surfaces:

$$
u=0 \text { and } v=\dot{y}(t)=a \omega \cos (\omega t)
$$

Symmetric boundary condition is applied to the axis of symmetry based on the axisymmetric flow assumption.

According to Thiagarajan \& Troesch (1998) based on their experimental observation, disturbance of the free surface is very small due to small amplitude and high frequency heave oscillation of the floating structures. Therefore, non-linear free surface effects are not included in the present numerical modelling, instead a free stream flow boundary condition is assumed, and pressure on the free surface is a constant. Free stream velocity conditions are also applied to the entire open boundary, and static pressure is assumed at far open boundaries. The Neumann boundary condition for the pressure on the cylinder and disk surfaces is obtained by applying the momentum equations in the direction normal to the body boundary.

\subsection{Hydrodynamic force coefficients}

The hydrodynamic heave force acting on the cylinder+disk is calculated by integrating the vertical component of the stress vector along the cylinder and disk surfaces.

$$
F=-\int_{B}\left\{-p n_{y}+\frac{1}{R_{e}}\left(\frac{\partial u}{\partial y}+\frac{\partial v}{\partial r}\right) n_{r}+\frac{2}{R_{e}} \frac{\partial v}{\partial y} n_{y}\right\} d s
$$


where $d s$ denotes the differential cylinder+disk surface area, $p$ the dynamic pressure, and $\left(n_{y}, n_{r}\right)$ the axial and radial components of the unit-normal vectors along the cylinder+disk surfaces. The three terms of the right hand side of the above expression represent the dynamic pressure component, the viscous shear-stress component and the normal viscous-stress component respectively. The viscous drag force on the body is first represented by an expression similar to the formulae of Morison's equation in which the drag is proportional to the square of the velocity. Since the cylinder and disk is subjected to a sinusoidal oscillation as shown in Eq. (9), the force can be linearised in a Fourier averaged sense,

$$
F(t)=-C_{a} \rho \forall \frac{\partial v}{\partial t}-C_{d} \frac{4}{3 \pi} \rho S V_{m}^{2} \cos (\omega t)
$$

where $V_{m}=a \omega$ is the amplitude of the velocity of the oscillation, and $T$ is the period of the oscillation. $S$ is the cross-section area of the disk, and $\forall$ is the immersed volume of the cylinder and disk. The drag and added mass coefficients are then obtained from a force time history by Fourier analysis, which gives:

$$
\begin{aligned}
& C_{d}=-\frac{3 \omega}{4 \varrho S V_{m}^{2}} \int_{0}^{T} F(t) \cos (\omega t) d t, \\
& C_{a}=\frac{1}{\pi \varrho \forall V_{m}} \int_{0}^{T} F(t) \sin (\omega t) d t,
\end{aligned}
$$

The equivalence between the Morison's equation-like formulae and the alternative expression $\left(F_{d}(t)=B v(t)\right)$ of the drag force by using the equivalent linear damping coefficient $B$ results in

$$
B=\frac{1}{3} \mu \beta D_{d}(K C) C_{d}
$$


where $\mu$ is the dynamic viscosity. In the analysis of the results, the equivalent linear damping coefficient $B$ is then normalised to give a non-dimensional damping ratio

$$
Z=\frac{B}{C_{c}}
$$

where $C_{c}=2\left(m+m_{a}\right) \omega$ is the critical damping of the system, $m$ and $m_{a}$ represent the mass and added mass respectively. By inserting expressions for the mass $m+$ $m_{a}=C_{m} \varrho \forall$ and $B$, one can obtain the damping ratio

$$
Z=\frac{1}{3 \pi^{2}} \frac{D_{d}^{2} D_{c}}{\left(D_{c}^{2} T_{d}+D_{d}^{2} t_{d}\right)} \frac{C_{d}}{C_{m}}(K C)
$$

where $D_{c}$ is the diameter of the cylinder while $T_{d}$ and $t_{d}$ represent the draft of cylinder and the thickness of the disk respectively.

Viscous shear stress acting on the cylinder+disk side wall in phase with velocity is the main source of friction damping force, form damping component is mainly attributed to the flow separation and vortex shedding at the sharp edges of the disk. Substituting the viscous shear stress term and the dynamic pressure term of Eq. (11) instead of total force $F(t)$ into Eq. (13), one can derive the two components of damping coefficient and damping ratio respectively.

\section{Numerical implementation}

The initial boundary value problem outlined above has fully non-linear field equations with boundary conditions. The theoretical formulation is implemented by using finite difference method based on curvilinear coordinates. The method is to solve the non-linear governing equations together with the given boundary conditions in primitive variable form. A non-staggered grid for the velocity and pressure 
is used in the present work.

\subsection{Spatial scheme}

The discretisation of the convection terms of the momentum equations is of primary concern due to its non-linear nature. This is particularly important for high Reynolds number flows since the convection is expected to be the dominant feature. In the present study, a modified second-order four-point upwind scheme (K-K scheme) proposed by Kawamura \& Kuwahara (1984) is used to discretise the nonlinear convection terms of the momentum equations,

$$
\begin{aligned}
\left(f \frac{\partial u}{\partial \xi}\right)_{i, j} & =\frac{f_{i, j}}{12 \triangle \xi}\left(-u_{i+2, j}+8 u_{i+1, j}-8 u_{i-1, j}+u_{i-2, j}\right) \\
& +\frac{\left|f_{i, j}\right|}{4 \triangle \xi}\left(u_{i+2, j}-4 u_{i+1, j}+6 u_{i, j}-4 u_{i-1, j}+u_{i-2, j}\right) .
\end{aligned}
$$

The second-order central differencing is used for all the other terms.

$$
\begin{aligned}
& \left(\frac{\partial f}{\partial \xi}\right)_{i, j}=\frac{f_{i+1, j}-f_{i-1, j}}{2} \\
& \left(\frac{\partial^{2} f}{\partial \xi^{2}}\right)_{i, j}=f_{i+1, j}+f_{i-1, j}-2 f_{i, j} .
\end{aligned}
$$

All the spatial terms in the transformed pressure Poisson equation are discretised by using second-order central differencing except those on the boundaries. The spatial derivatives in the boundaries are discretised by a second-order one-sided differencing, i.e. for left boundary, we have,

$$
\left(\frac{\partial f}{\partial \xi}\right)_{i, j}=\frac{-3 f_{i, j}+4 f_{i+1, j}-f_{i+2, j}}{2}
$$




$$
\left(\frac{\partial^{2} f}{\partial \xi^{2}}\right)_{i, j}=f_{i+2, j}-2 f_{i+1, j}+f_{i, j}
$$

Similar spatical derivatives were derived for all the other boundaries.

\subsection{Time integration}

The momentum Eqs (5) and (6) are marched forward in time by using a secondorder fully implicit scheme (Beaudan, 1994):

$$
\frac{3 \mathbf{V}^{n+1}-4 \mathbf{V}^{n}+\mathbf{V}^{n-1}}{2 \Delta t}+\mathbf{V}^{n+1} \cdot \nabla \mathbf{V}^{n+1}=-\nabla p^{n}+\frac{1}{R_{e}^{\prime}} \nabla^{2} \mathbf{V}^{n+1}
$$

The primitive variables of velocity and pressure are obtained by solving momentum equations and pressure Poisson equation alternatively using an iterative method. The discrete form of nonlinear Eq. (23) is first solved by using the Gauss-Seidel iterative method with pressure at time level $(n)$. A solution for pressure at time level $(n+1)$ is then obtained by solving the discrete form of pressure Poisson equation using Successive Over-Relaxation (SOR) scheme. The correction term

$\left(\frac{\partial \tilde{D}}{\partial t}\right)$ in the pressure Poisson equation is discretised with the second-order timeaccurate scheme.

The governing equations together with the given boundary conditions are solved as an initial boundary value problem. It is assumed that the flow induced by the heaving cylinder starts from a quiescent state, i.e. velocity is zero at time $t=0$. 


\subsection{Mesh generation and numerical parameters}

Due to the axisymmetric flow assumption, meshes for the simulation of the flow induced by the oscillating cylinder and disk are generated in a rectangular region simply by an algebraic method. Although interference of the shear layer developed along cylinder surface to the vortex shedding is expected as amplitude of the oscillation increases, since the amplitudes of oscillations are relatively small, the vortex patterns around the cylinder+disk mainly depend on the local flow around the sharp edges of the disk. Based on these considerations, a mesh is then constructed in such a way that the node points are concentrated near the cylinder and disk surfaces and stretched out gradually. As can be seen from Fig. 3, the grid is fully orthogonal, and of high quality from the mesh generation point of view. The small regions around the sharp edges of the disk, where the vortices are generated and shed during the oscillation, are emphasised with clustering the grid points to the regions. Such a grid distribution ensures both high quality computation in the vicinity of sharp edges and global efficiency.

The size of flow domain and the numerical parameters $\Delta r, \Delta y, \Delta t$ as well as the number of nodes necessary for the simulation of the flow generated by the oscillating cylinder and disk, are evaluated in order to ensure the stability of the computations and to minimize the numerical error for each value of the flow parameters. Based on the extensive numerical tests of (Tao et al., 2000; Tao, 2002), the radial and bottom open boundaries of the flow domain are located at $10 R$ from the cylinder wall and bottom surface respectively. A mesh of $(134 \times 120)$ with a minimum grid spacing of $0.0005 R$ around the sharp edges of the disk in both radial and vertical directions are used in the numerical simulations. All the calculations are carried out with a time step $\Delta t / T=1 / 5000$. The mesh is regenerated at each time step 
according to the forced oscillation of cylinder+disk.

\section{Validation of numerical method}

The first principal step taken to validate the present numerical procedure is the convergence tests with varying flow domains and mesh parameters (Tao, 2002). In this section, the present numerical procedure is further validated against twodimensional benchmark problem: lid-driven flow in a square cavity, and comparisons with the experimental results of Thiagarajan \& Troesch (1994) for the flow of an oscillating truncated vertical cylinder.

\subsection{Lid-driven flow in a square cavity}

The lid-driven flow in a square cavity is a typical steady separated flow which has been examined experimentally and numerically in the past (Burggraf, 1966; Bozeman \& Dalton, 1973; Schreiber \& Keller, 1983)). Accurate numerical results are available for this case over a certain range of Reynolds number which is defined in terms of the driven velocity at the top and the width of the cavity. Therefore, it has been frequently used to test the numerical solutions of the Navier-Stokes equations. The problem definition and the flow pattern in the cavity are illustrated schematically in Fig. 4. The flow is characterised by a strong primary vortex generated by the top boundary (lid) in the upper portion of the cavity. In addition, there is a pair of small counter-rotating secondary vortices of very low strength located in the lower corners of the cavity. The location of the primary vortex and the velocity distributions in the cavity depend on Reynolds number.

The comparison of the centre locations $\left(X_{c}, Y_{c}\right)$ of the primary vortex obtained 
Table 1

Locations of the primary vortex centre

\begin{tabular}{c|ccc|cccc}
\hline $\operatorname{Re}$ & \multicolumn{2}{|c|}{ Schreiber \& Keller $(1983$} & \multicolumn{3}{|c}{ Present } \\
& & & & \multicolumn{3}{c}{} \\
& Mesh size & $X_{c}$ & $Y_{c}$ & Mesh size & $X_{c}$ & $Y_{c}$ \\
\hline 40 & $121 \times 121$ & 0.56667 & 0.75833 & $81 \times 81$ & 0.55898 & 0.74900 \\
100 & $121 \times 121$ & 0.61667 & 0.74167 & $81 \times 81$ & 0.62393 & 0.74413 \\
400 & $141 \times 141$ & 0.55714 & 0.60714 & $81 \times 81$ & 0.55478 & 0.60265 \\
1000 & $141 \times 141$ & 0.52857 & 0.56429 & $81 \times 81$ & 0.53307 & 0.56744 \\
\hline
\end{tabular}

from the present numerical calculation and the results of Schreiber \& Keller (1983)

in Table 1 shows very good agreement with maximum difference of $1.36 \%$. Fig. 5 presents the distributions of the horizontal velocity component along the vertical lines at $x=0.1,0.5$ and 0.9 , obtained from the present numerical solution and Schreiber \& Keller (1983) at Reynolds number $R e=1000$. As can be seen in Fig. 5 , although a relatively coarse mesh is applied in the present calculations, excellent agreement has been achieved.

\subsection{Flow generated by an oscillating truncated vertical cylinder}

Several researchers investigated the flow generated by a truncated vertical cylinder oscillating at low Keulegan-Carpenter numbers, corresponding to the hydrodynamic phenomenon 'springing' of deep water offshore structure TLPs (Chakrabarti, 1991; Huse, 1990; Huse \& Utnes, 1994; Thiagarajan \& Troesch, 1994). Experimental data available from Thiagarajan \& Troesch (1994) is the main reference for validating the present numerical investigation. Forced oscillation experiments 
were performed with a circular cylinder of $0.457 \mathrm{~m}$ diameter and $1.219 \mathrm{~m}$ draft. The cylinder was oscillated corresponding to $K C$ from 0.1 to 1.0. The frequency parameter $\beta$ is 89236 , corresponding to the natural frequency of the cylinder of $0.41 \mathrm{~Hz}$. Since the total force is inertia dominated, Thiagarajan \& Troesch (1994) conducted damping measurements on cylinders in forced oscillation at or near resonance, in witch the restoring forces nearly offset the system inertia forces. The resulting force measurements give a good estimate of the drag coefficients.

A rectangular flow domain of $11 R \times 15 R$ with a mesh $88 \times 93$ is used in the calculations. The damping ratio for a single cylinder can be derived as

$$
Z_{c}=\frac{1}{3 \pi^{2}} \frac{D}{T_{d}} \frac{C_{d}}{C_{m}}(K C)
$$

Fig. 6 is a plot of the damping ratio $Z_{c}$ calculated by using Eq. (24) and the experimental results of Thiagarajan \& Troesch (1994). The friction drag component of the damping ratio of the present numerical estimation $Z_{\text {friction }}$ and that calculated based on the laminar boundary theory are also plotted in Fig. 6. As can be observed from the figure, the numerical solutions agree reasonably well with the experimental results. Damping ratio of both numerical solution and experimental data showed approximately linear trends in the range of $K C=0.1 \sim 1.0$.

Applying laminar boundary layer theory to a vertical cylinder of radius $R$ and draft $T_{d}$ in heave, the friction drag component, i.e. the shear force in phase with velocity, of the damping coefficient and damping ratio are obtained as:

$$
\begin{aligned}
& B_{\text {friction }}=\pi^{1.5} T_{d} \mu \beta^{0.5} \\
& Z_{\text {friction }}=\frac{2}{C_{m}}\left(\frac{1}{4 \pi \beta}\right)^{0.5} .
\end{aligned}
$$

Eqs. (25) and (26) show that $B_{\text {friction }}$ and $Z_{\text {friction }}$ are independent of $K C$ based 
on laminar boundary layer theory.

The following observations were made regarding the friction drag of the present numerical results and that calculated using Eq. (25) shown in Fig. 6. Firstly, $Z_{\text {friction }}$ of the present numerical solution is approximately independent of $K C$, and about $7 \%$ higher than the value calculated from laminar boundary layer theory. This agreement can be regarded as acceptable. The additional friction drag may be attributed to the turbulence effect although $K C$ is very small. Secondly, $Z_{\text {friction }}$ tends to decrease slightly with increasing $K C$ due to the leading edge effect of the truncated cylinder, while Eqs. (25) and (26) are based on the infinite length cylinder. Tao et al. (2000) revealed that the effect of the leading edge depends on the aspect ratio (diameter/draft) of the cylinder. In the present case of the aspect ratio about 0.35 , numerical computation shows that $Z_{\text {friction }}$ drops about $8 \%$ as $K C$ increases from 0.001 to 1.0 at $\beta=89236$.

As shown in Fig. 6, numerical estimation of the damping ratio $Z$ is approximately linear with $K C$ in the range of $0.045 \sim 1.0$ and appears to be a constant as $K C \rightarrow$ 0 at $\beta=89236$. By subtracting the $K C$ independent friction component, the form drag component of damping ratio which contributes to the slope of the curves also shows approximately linear trend with $K C$ in the range of $0.045 \sim 1.0$, and tends to be constant as $K C \rightarrow 0$.

Fig. 6 also shows that the form drag component of the damping ratio of the numerical results appears to be in general larger than the experimental data. In the present numerical investigation, cylinder bottom edge is at 90 degrees, but this may be hard to obtain in an experimental setup. Tao \& Thiagarajan (2000) demonstrated that a very small corner radius of the bottom edge can result in a significant reduction of the form drag. 


\section{Vortex shedding and hydrodynamics of oscillating cylinder+disk configu- ration}

Tao (2002) have conducted numerical study on the effect of the oscillation parameter $(K C)$ and geometry, primarily the thickness of the disk for a cylinder+disk configuration on the behaviour of the vortex shedding. As a result of his comprehensive study, three vortex shedding modes were observed for a parameter range of $K C=0.00075 \sim 0.75$ and aspect ratio $t_{d} / D_{d}=0.00075 \sim 1.642$. The enhancement on vortex shedding due to reducing thickness of the disk was identified. However, a systematic variation of disk geometry, especially the diameter of the disk and the corresponding influence on damping and added mass was not fully explored. Further, current practice in offshore engineering design of heave plate for Spar or truss spar platform is based on model tests which were conducted at low $\beta$ and the recommended drag coefficient for plates was between 2 and 3. However, there could be a significant difference in drag coefficient between model test results and prototypes (Prislin et al., 1998). The focus of this study is on the effect of the diameter ratio of the cylinder+disk configuration on heave damping and added mass. Flow of oscillating cylinder with a disk attached to its base is calculated for a broad range of diameter ratio, $D_{d} / D_{c}$, from 1.0 to 2.0. The vortex shedding patterns are presented for various values of $D_{d} / D_{c}$. Finally, the optimum diameter ratio in terms of heave motion suppression is determined based on the heave damping calculation. The influence of Reynolds number (and consequently $\beta$ ) on the hydrodynamic damping is also investigated by examining the damping behaviour in realistic $\beta$ in a case study.

In order to illustrate the development of the vortex shedding pattern, the vorticity in a meridional plane is computed by taking the curl of the velocity field. In the 
axi-symmetrical case,

$$
\zeta=\frac{\partial u}{\partial y}-\frac{\partial v}{\partial r}
$$

\subsection{Influence of diameter ratio on vortex shedding pattern}

Fig. 7 show vorticity contours of the flow around the disk edges due to cylinder+disk oscillating at $K C=1.0$ computed from Eq. (27). The instantaneous vorticity contours presented in Fig. 7 are equally spaced over one cycle. The vortices formed with a smaller disk diameter (Fig. 7(a-d)) appear compressed showing the influence of the presence of the cylinder wall. On the other hand, vortices at larger disk diameter (Fig. 7(e-h)) are more rounded and appear to move around without the cylinder's hindrance. From the point of view of energy conservation, vortices leaving a control volume surrounding the cylinder and disk represents effective means of energy dissipation and therefore imply increased damping forces due to stronger vortex shedding processes at larger disk diameter. According to Thiagarajan et al. (2002), the core diameter is of the same order of the oscillation stroke. Therefore, the disk extension should be at least of the same order being sufficient to accommodate the core of the vortex ring shed during an oscillation process.

\subsection{Influence of geometry on the hydrodynamic coefficients}

In order to compare the influence of the disk geometry on the hydrodynamic coefficients of oscillating cylinder+disk configuration at same amplitude of oscillation, a new Keulegan-Carpenter number and frequency parameter based on the diameter 
of cylinder are defined as

$$
\begin{aligned}
& K C_{c}=\frac{2 \pi a}{D_{c}} . \\
& \beta_{c}=\frac{\left(D_{c}\right)^{2} f}{\nu}
\end{aligned}
$$

\subsubsection{Effects of disk diameter on added mass coefficient}

The added mass of a disk oscillating along its axis approximately equal to the mass of a sphere of water enclosing the disk (Sarpkaya \& Isaacson, 1981)

$$
m_{a}=\frac{1}{3} \rho D_{d}^{3}
$$

For the configuration of a cylinder with a disk attached to its base, if the diameter of the disk is greater than that of the cylinder, there is only a part of the disk on the cylinder side producing added mass effect since the presence of the cylinder (see Fig. 8). Thus, the added mass of a cylinder+disk becomes

$$
\begin{aligned}
m_{a} & =\frac{1}{3} \rho D_{d}^{3}-\left[\frac{\pi \rho}{8} D_{c}^{2}\left(D_{d}-\sqrt{D_{d}^{2}-D_{c}^{2}}\right)\right. \\
& \left.+\frac{\pi \rho}{24}\left(D_{d}-\sqrt{D_{d}^{2}-D_{c}^{2}}\right)^{2}\left(2 D_{d}+\sqrt{D_{d}^{2}-D_{c}^{2}}\right)\right] .
\end{aligned}
$$

The added mass coefficient, $C_{a}^{\prime}$, of a cylinder+disk is then obtained as the ratio of the added mass to the displaced mass $\left(m+m_{a}\right)$

$$
\begin{aligned}
C_{a}^{\prime}= & \frac{1}{D_{d}^{2} t_{d}+D_{c}^{2} T_{d}}\left\{\frac{4}{3 \pi} D_{d}^{3}-\left[\frac{1}{2} D_{c}^{2}\left(D_{d}-\sqrt{D_{d}^{2}-D_{c}^{2}}\right)\right.\right. \\
& \left.\left.+\frac{1}{6}\left(D_{d}-\sqrt{D_{d}^{2}-D_{c}^{2}}\right)^{2}\left(2 D_{d}+\sqrt{D_{d}^{2}-D_{c}^{2}}\right)\right]\right\}
\end{aligned}
$$

Fig. 9 shows the results for added mass coefficient versus the diameter ratio $D_{d} / D_{c}$.

Excellent agreement between the results of the present numerical computation and 
theoretical expression demonstrated the applicability of Eq. (32) for calculating added mass coefficient for a cylinder+disk configuration. The little variation among the results from numerical calculation at three different $K C$ numbers shown in Fig. 9 indicates a weak influence of $K C$ on added mass.

Typically a classic Spar platform has a natural heave period around $25 \sim 30$ seconds, which in most circumstances is sufficiently outside the prevailing wave frequency range and thus heave motion is generally insignificant. However, a long swell condition, with peak period lying in the $23 \sim 25$ second range may persist a considerable portion of the year in West African offshore. Possible resonance with long period swell may result in large amplitude heave oscillations, and causes further damage to risers and mooring systems, as demonstrated in the model tests by Thiagarajan et al. (2002). For a floating vertical cylinder, the heave natural period of the cylinder can be derived from dynamic analysis as

$$
T=2 \pi \sqrt{\frac{m+m_{a}}{\rho g A+k_{m}}}
$$

where $A$ denotes the area of water plane, and $k_{m}$ is the mooring stiffness. For a freely floating cylinder of uniform cross section area without mooring effects, Eq. (33) is simplified to

$$
T=2 \pi \sqrt{\frac{T_{d}\left(1+C_{a}\right)}{g}}
$$

where $T_{d}$ is the draft of the cylinder. Eqs (33) and (34) indicate that the natural heave period increases on increasing added mass. Thus, the longer natural heave period of a Spar by increasing the diameter of the disk is favourable in preventing serious occurrence of heave resonance. However, extension of this approach to another type of deep water offshore structure TLPs should be done with caution, since 
increasing the natural heave period by a disk of increasing diameter, from very low value, typically $2 \sim 5$ seconds, may result in closer to the peak period of a wave spectrum, and further amplifying springing vibration.

\subsubsection{Effects of disk diameter on damping ratio}

As observed in flow visualisation, the diameter of the disk has a significant influence on vortex shedding patterns. Since form damping dominant, the most basic and typical diameter ratio effects on vortex shedding behaviour of the cylinder+disk flow can also be investigated by examining the diameter ratio effects on the hydrodynamic damping, and this is certainly of more interests in offshore engineering design. In Fig. 10, the viscous damping ratio, as a function of diameter ratio $D_{d} / D_{c}$ for three $K C_{c}$ numbers, $0.1,0.5$ and 1.0 are plotted. The curves in Fig. 10 show a weak nonlinear trend of $Z$ with $D_{d} / D_{c}$ at different $K C_{c}$. The damping ratio appears to increase as diameter ratio increases for all three $K C_{c}$ numbers. However, considerable flattening of the curves are seen at larger disk diameters. As observed in flow visualisation, for small diameter ratio, since the small extension of the disk edges relative to the cylinder wall, interference of the cylinder boundary on the vortex formation and shedding process is evident, even at small $K C_{c}$ number. The vortices formed with a disk of smaller diameter appear suppressed, resulting in lower damping. As the diameter of the disk increases to certain stage, the extension is much larger than the length scale of the vortex formation primarily dependent on $K C_{c}$, the interference of the cylinder boundary does not occur. The vortex shedding process around the disk edges becomes independent of diameter ratio, indicating that any further increase in the disk diameter would not result in appreciable increases in drag. It is also seen in the figure that the slope of the curve increases on higher $K C_{c}$ number, and tends to flatten at higher diameter ratio due to the interference 
from the cylinder to the larger vortices generated. Therefore, the stronger diameter ratio dependence of viscous damping is only significant in combination with $K C$ number effects. In realistic offshore situation, the amplitude of second-order "springing" vibration of a TLP is very small, corresponding to $K C$ number in the order 0.01 or less, while the $K C$ number of possible heave resonance for a Spar can be as high as 1.0. Therefore, different diameter extensions should be selected based on the above argument. For the highest $K C$ of 1.0 in Fig. 10, the flatterning occurs at $D_{d} / D_{c} \approx 1.65$, corresponding to an extension of the disk approximately four times the oscillation stroke.

\subsubsection{Effects of $K C$}

Fig. 11 shows the variation of damping ratio, $Z$, against $K C_{c}$ at $\beta_{c}=89236$. Again, cases considered here include diameter ratio range from 1.0 to 2.0 , being representative for flow generated by a single cylinder and a cylinder with disks of small to large diameter ratio respectively. Two components of viscous damping ratio, i.e. friction drag $\left(Z_{\text {friction }}\right)$ and form $\operatorname{drag}\left(Z_{\text {form }}\right)$ for $D_{d} / D_{c}=1.33$ are also plotted in Fig. 11. As shown in the figure, over the range of $K C_{c}$ calculated, friction damping is approximately constant. This indicates that friction damping is independent of amplitude of the oscillation. However, form damping shows predominant and increases dramatically as $K C_{c}$ increases except for extremely low $K C_{c}$, in which form damping is approaching a constant value as $K C_{c} \rightarrow 0$, since separation may not occur or very weak, resulting in very small form damping. Similar trend of viscous damping was also observed for a single truncated cylinder and cylinder+disk at $D_{d} / D_{c}=2.0$. Depending on the diameter ratio, all curves of $Z \sim K C_{c}$ show that the non-linear relationship exists between the damping ratio and $K C_{c}$. 


\subsubsection{Effects of disk thickness $t_{d} / D_{d}$}

As observed in flow visualisation, a fully developed vortex shedding process and increasing vorticity formed around the disk due to lack of interference from the cylinder boundary layer at a larger diameter ratio, indicate enhancing energy dissipation. As show in Fig. 10, this further results in the significant increase in heave damping. Damping variation due to different aspect ratio, $t_{d} / D_{d}$, is shown in Fig. 12. As can be seen from the figure, the increase in damping ratio is steeper when the disk is thinner. This is due to the absence of disk thickness significantly enhances interaction of the vortices formed during any two successive half cycles of the flow leading to increased shed vorticity. However, the trend of increasing heave damping for the thinner disk is reversed for aspect ratio around $0.12 \sim 0.16$, probably due to the forces of viscosity and convection cancelling each other in the small range. Therefore, to maximise the damping effects, minimum thickness that satisfies structural and fatigue strength requirements should be chosen.

\section{Case study}

As shown in Fig. 11, the heave damping comprises of two major components, i.e. small and $K C$ independent friction drag and predominant $K C$ dependent form drag. This is a clear indication that flow separation and vortex shedding are the main mechanism of energy dissipation. Therefore, one could argue the influence of $\beta$, and consequently Reynolds number to be a minimum. The hydrodynamic behaviour of a Spar prototype with a heave plate oscillating at realistic $\beta$ number of $10^{7}$ will be examined through the calculation of viscous damping and added mass using the present numerical method. 
Table 2

Oscillation parameters and geometry of prototype Spar and heave plate

\begin{tabular}{|c|c|c|c|c|c|c|}
\hline Configuration & 1 & 2 & 3 & 4 & 5 & 6 \\
\hline Spar diameter $(\mathrm{m})$ & \multicolumn{6}{|c|}{39.0} \\
\hline Total draft (m) & \multicolumn{6}{|c|}{198.1} \\
\hline Disk thickness (m) & no disk & 0.475 & 0.475 & 0.475 & 0.475 & 0.475 \\
\hline Disk diameter (m) & no disk & 42.1 & 45.1 & 51.2 & 61.0 & 78.0 \\
\hline Diameter ratio $\left(D_{d} / D_{c}\right)$ & 1.0 & 1.08 & 1.16 & 1.31 & 1.56 & 2.0 \\
\hline Aspect ratio $\left(t_{d} / D_{d}\right)$ & no disk & 0.0113 & 0.0105 & 0.0093 & 0.0078 & 0.0061 \\
\hline Amplitude (m) & \multicolumn{2}{|c|}{0.91} & \multicolumn{2}{|c|}{2.74} & \multicolumn{2}{|c|}{4.57} \\
\hline$K C$ & \multicolumn{2}{|c|}{0.15} & \multicolumn{2}{|c|}{0.44} & \multicolumn{2}{|c|}{0.74} \\
\hline Period (sec) & \multicolumn{6}{|c|}{28} \\
\hline$\beta$ & \multicolumn{6}{|c|}{$4.57 \times 10^{7}$} \\
\hline
\end{tabular}

Numerical simulations were conducted with a $198.1 \mathrm{~m}$ draft and $39.0 \mathrm{~m}$ diameter Spar column and a $0.475 \mathrm{~m}$ thick disk and five different disk diameters of 42.1 $\mathrm{m}, 45.1 \mathrm{~m}, 51.2 \mathrm{~m}, 61.0$ and $78.0 \mathrm{~m}$. Six configurations comprising of the bare hull and five different disk diameters, oscillating at three different amplitudes of oscillation at a constant period of $28 \mathrm{sec}$ were considered. The corresponding $K C_{c}$ and $\beta$ numbers were $0.15,0.44,0.74$ and $4.6 \times 10^{7}$ respectively (see Table 2 ).

The behaviour of $Z$ as a function of the diameter ratio is shown in Fig. 13. Shown also in the figure are the experimental values obtained by Thiagarajan et al. (2002) for low amplitudes and high amplitudes of oscillation at $1: 75$ model scale. It is 
noted that while the computational problem is for forced heave oscillation, the experimental method is based on heave decay oscillations, where amplitude changes with time. In spite of this difference in approaches, the agreement shown in Fig. 13 is remarkably good, for both low and high $K C$ regimes. The significance of the good agreement is the fact that the computational results were obtained for realistic $\beta$ numbers typical of prototype values, while the $\beta$ numbers in model test are several orders smaller than prototypes. This is a clear indication that $\beta$ effects in estimation of heave damping can be considered negligible, since most of damping is geometry related form damping due to flow separation and vortex shedding. Similar to the results obtained from the calculations at model scale, the curves in Fig. 13 show a weak nonlinear trend of $Z$ with $D_{d} / D_{c}$ at different $K C_{c}$. Considerable flattening of the curves is seen when the disk diameter gets larger due to the diminish of the interference between the vortex shedding and Spar hull. For this particular Spar prototype, $Z$ is found to be approximately constant beyond $D_{d} / D_{c}=1.16$ at $K C_{c}=0.15$, corresponding to a disk of diameter $45.2 \mathrm{~m}$, i.e. an extension of $3.1 \mathrm{~m}$ to Spar hull. This value is about three times of the amplitude. However, at seastates with wave spectrum of long peak period, such as West Africa, a classic Spar can suffer from severe heaving with $K C_{c}$ up to $0.7 \sim 1.0$. As can be seen from Fig. 13, the flattening trend of the curves for larger $K C_{c}$, i.e. 0.44 and 0.74 , starts at much larger diameter ratio values, approximately 1.3 and 1.6 respectively. Therefore, consideration of $K C$ number range of the oscillation must be taken when one to determine the disk geometry for heave suppression. For instance, the heave oscillation of a Spar may be rather large ( $K C_{c}$ up to 1.0) while the amplitude of a TLP "springing" is very small $\left(K C_{c}<0.01\right)$. As a general guideline it is suggested that the disk extension be at least four times the typical heave amplitude to get the optimum drag effect. 


\section{Conclusions}

An axisymmetric finite difference method has been validated for the prediction of the flow generated by an oscillating vertical cylinder with a circular disk attach to its base at low Keulegan-Carpenter numbers. The numerical scheme has been used to investigate the vortex shedding flow and the associated hydrodynamic behaviour of the cylinder+disk configuration. The dependence of vortex shedding pattern on the diameter ratio $D_{d} / D_{c}$ and $K C$ number have been accurately predicted.

The strong influence of diameter ratio was found on the vortex shedding and the viscous damping. However, the effect of changing diameter ratio was found to be dependent very strongly on $K C$ number. Within the parameter range of $K C$ up to 1.0 calculated in this study, it is found that the form drag due to flow separation and vortex shedding generated by the sharp edges of the disk are the dominant mechanism of energy dissipation.

Selection of the disk geometry in terms of the diameter should be considered in combining with $K C$ number of heave oscillation since the disk geometry effect on drag is strongly influenced by $K C$ number. A general trend of increasing damping ratio on increasing diameter ratio is found from the present numerical calculation. However, any increase beyond a certain diameter would not result in appreciable increases in drag. Based on the present study, it is suggested that the disk extension be at least four times the typical heave amplitude to get the optimum drag effect.

Case study further demonstrated that $\beta$ effects in estimation of heave damping can be considered negligible, since most of damping is geometry related form damping due to flow separation and vortex shedding. The results obtained from this study can be directly applied to the design of heave plate of floating offshore structures 
such as TLP, Spar and truss spar.

\section{References}

Beaudan, P. \& Moin, P.1994 Numerical experiments on the flow past a circular cylinder at sub-critical Reynolds number, Report No. TF-62, Department of Mechanical Engineering, Stanford University.

Bozeman, J. D. \& Dalton, C. 1973 Numerical study of viscous flow in a cavity. Journal of Computational Physics 12, 348-363.

Burggraf, O. R. 1966 Analytical and numerical studies of the structure of the steady separated flows. Journal of Fluid Mechanics 24, 113-151.

Chakrabarti, S. K. \& Hanna, S.Y. 1991 High-Frequency Hydrodynamic Damping of a TLP Leg. Proceedings of 10th International Offshore Mechanics and Arctic Engineering 1-A, 147-151.

Huse, E. 1990 Resonant Heave Damping of Tension Leg Platforms. Proceedings 22nd Annual OTC Paper 6317, 431-436.

Huse, E. \& Utnes, T. 1994 Springing Damping of Tension Leg Platforms. Proceedings 26nd Annual OTC Paper 7446, 259-267.

Kawamura, T. \& Kuwahara, K. 1984 Computation of High Reynolds Number Flow around a Circular Cylinder with Surface Roughness. AIAA Paper No. 84-0340, $1-11$.

Prislin, I., Blevins, R.D. \& Halkyard, J. 1998 Viscous damping and added mass of solid square plates. Proceedings of The 17th International Conference on Offshore Mechanics and Arctic Engineering.

Sarpkaya, T. \& Isaacson, M. 1981 Mechanics of Wave Forces on Offshore Structures., van Nostrand Reinhold, New York. 
Schreiber, R. \& Keller, H. B. 1983 Driven cavity flows by efficient numerical techniques. Journal of Computational Physics 49, 310-333.

Tao, L., Thiagarajan, K. P. \& Cheng, L. 2000 On the parametric dependence of springing damping of TLP and Spar columns. Applied Ocean Research 22, 281-294.

Tao, L. \& Thiagarajan, K. P. 2000 The Influence of Edge Sharpness on the Heave Damping Forces Experienced by a TLP Column. Proceedings of The 10th International Offshore and Polar Engineering Conference (ISOPE) I, 277-282.

Tao, L. 2002 Numerical Investigation of Hydrodynamic Heave Damping of Deep Water Offshore Structures, Ph.D. Thesis, The University of Western Australia.

Thiagarajan, K. P. 1993 Hydrodynamics of Oscillating Cylinder and Disks at Low Keulegan-Carpenter Numbers, Ph.D. Thesis, Department of Naval Architecture and Marine Engineering, The University of Michigan.

Thiagarajan, K. P. \& Troesch, A. W. 1994 Hydrodynamic heave damping estimation and scaling for tension leg platforms Columns. Journal of Offshore Mechanics and Arctic Engineering, ASME 116, 70-76.

Thiagarajan, K. P. \& Troesch, A. W. 1998 Effects of Appendages and Small Currents on the Hydrodynamic Heave Damping of TLP Columns. Journal of Offshore Mechanics and Arctic Engineering, ASME 120, 37-42.

Thiagarajan, K. P. Datta, I., Ran, Z., Tao, L. \& Halkyard, J. 2002 Influence of heave plate geometry on the heave response of classic Spars. Proceedings of The 21st International Conference on Offshore Mechanics and Arctic Engineering OMAE2002-28350. 


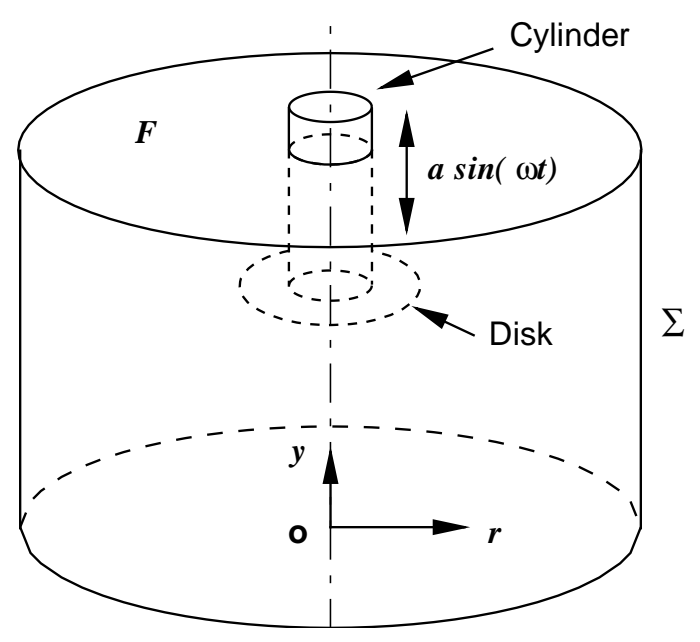

Fig. 1. Flow configuration for a cylinder with a disk attached on its bottom in heave

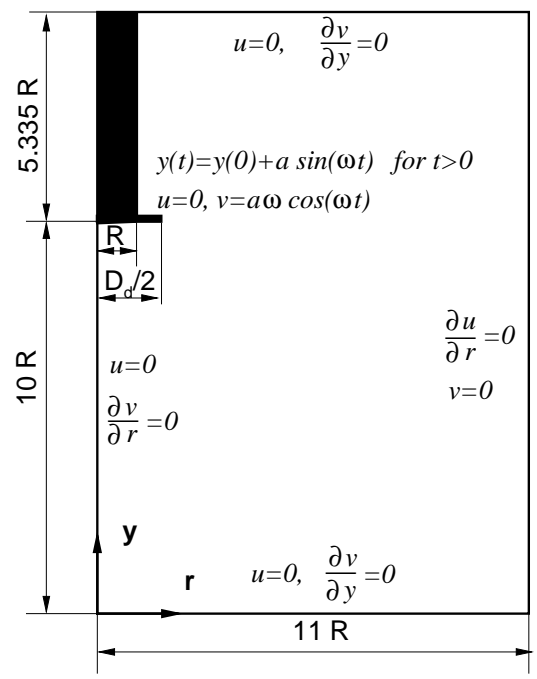

Fig. 2. Flow domain and boundary conditions 

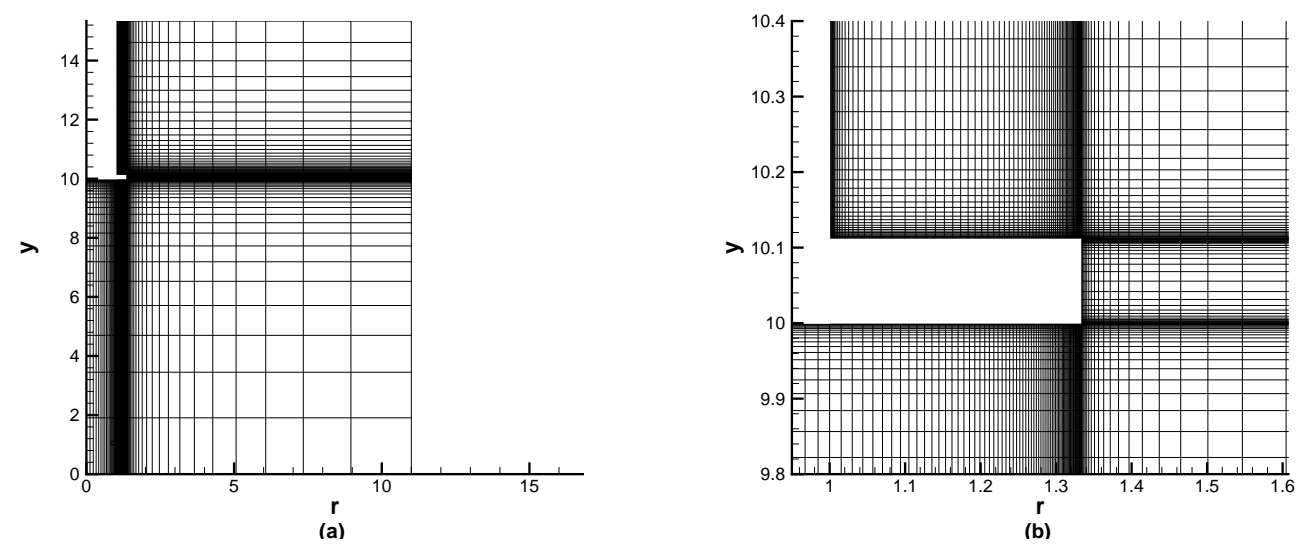

Fig. 3. Mesh generation for the simulation of flow induced by an oscillating cylinder: (a) Physical grid; (b) Typical grid distribution around the disk surface.

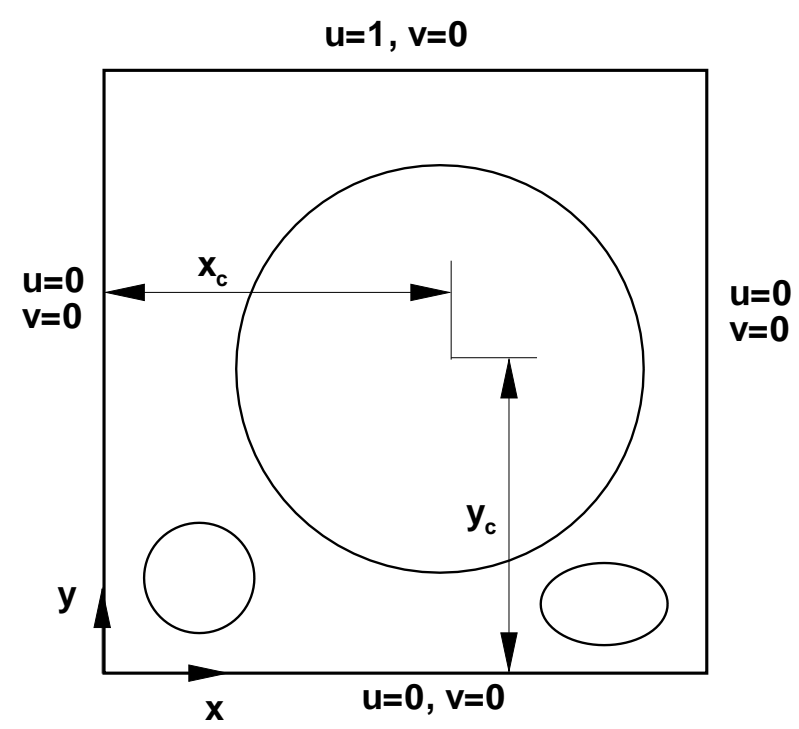

Fig. 4. Problem definition and flow pattern of a lid-driven square cavity 


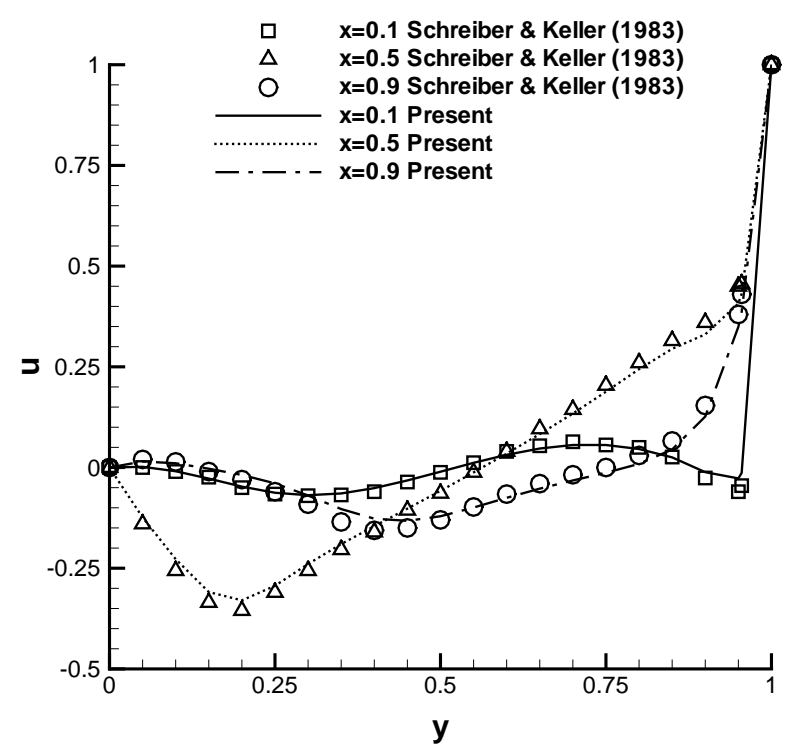

Fig. 5. Distributions of the horizontal velocity component of a lid-driven square cavity at $R e=1000$

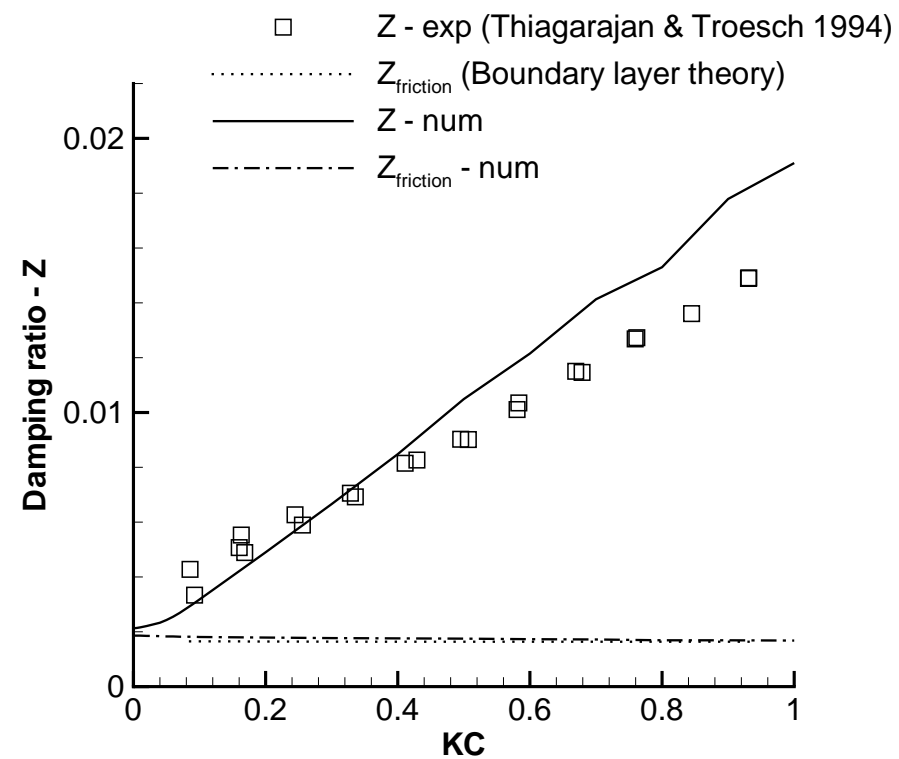

Fig. 6. Damping ratio $Z$ versus $K C$ at $\beta=89236$. 


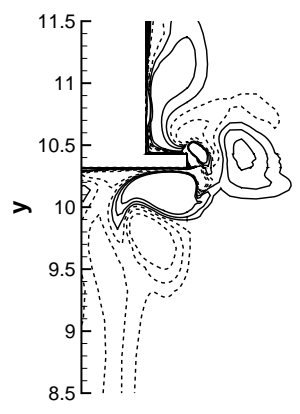

(a)

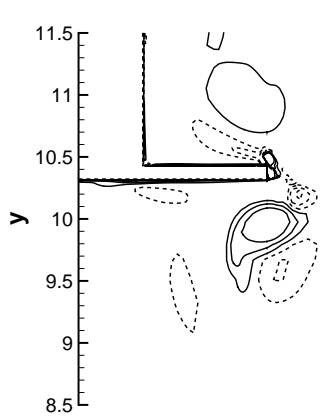

(e)

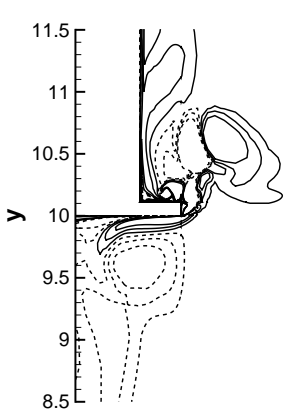

(b)

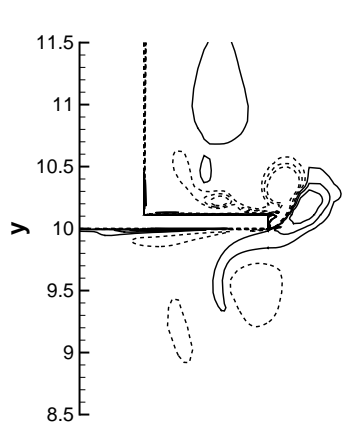

(f)

(c)
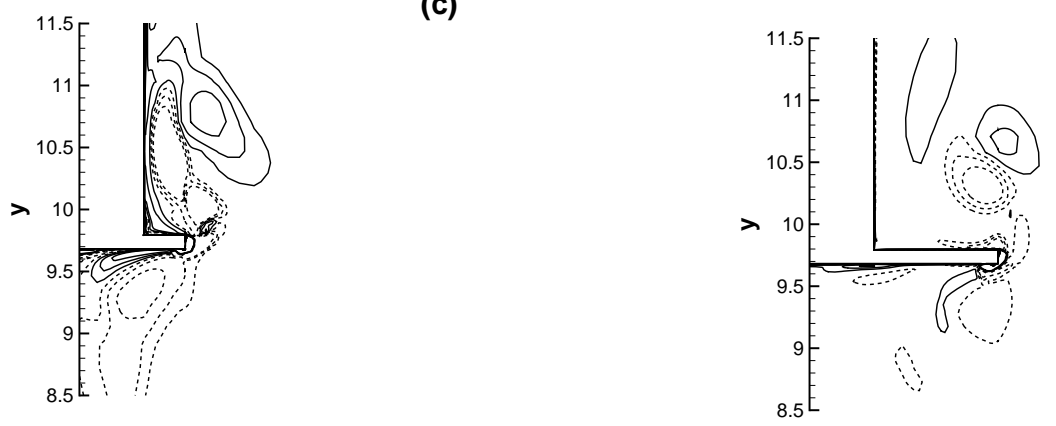

(g)

(d)

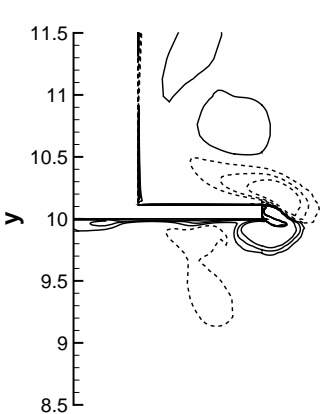

(h)

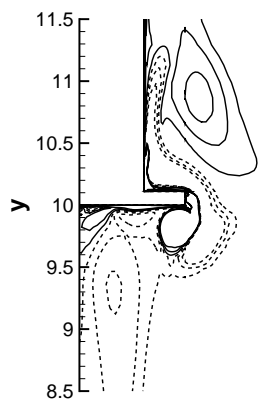

Fig. 7. Vortex formation around the disk at $K C_{c}=1.0, \beta_{c}=89236$. (a) to (d) represent flow for $D_{d} / D_{c}=1.33$ and (e) to (h) represent flow at $D_{d} / D_{c}=2.0$. Dashed lines indicate negative vorticity. 


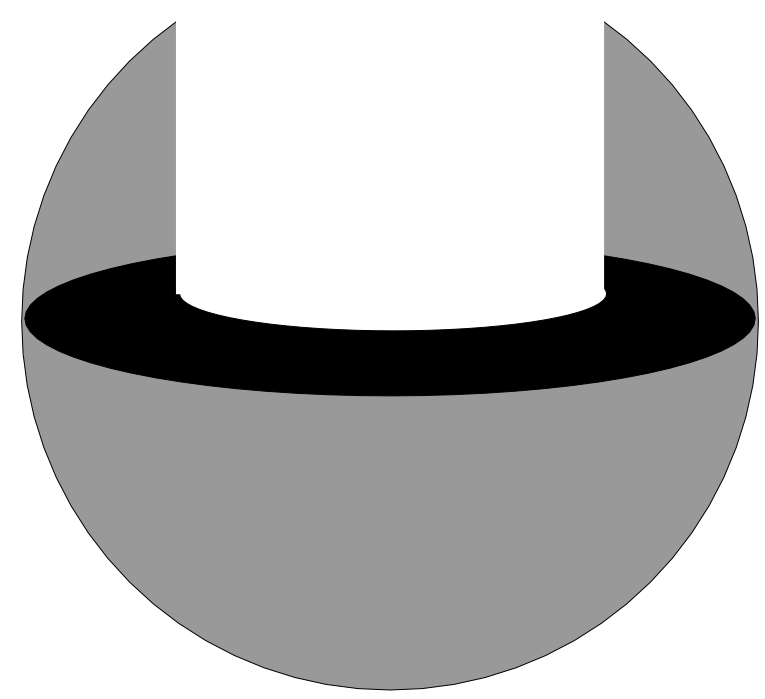

Fig. 8. Added mass of a disk attached to a cylinder

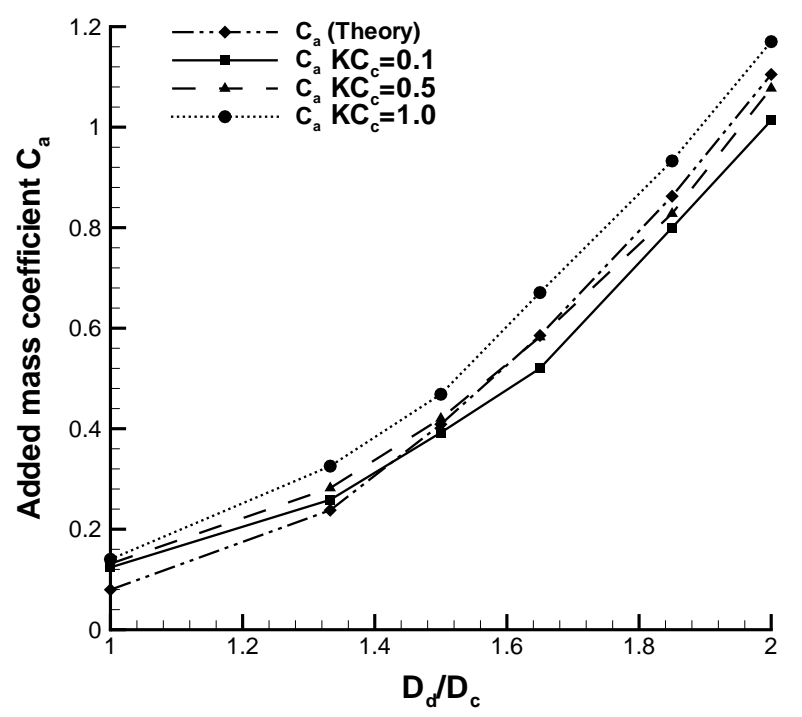

Fig. 9. Added mass coefficient $C_{a}$ versus $D_{d} / D_{c}$ at different $K C_{c}\left(\beta_{c}=89236\right)$ 


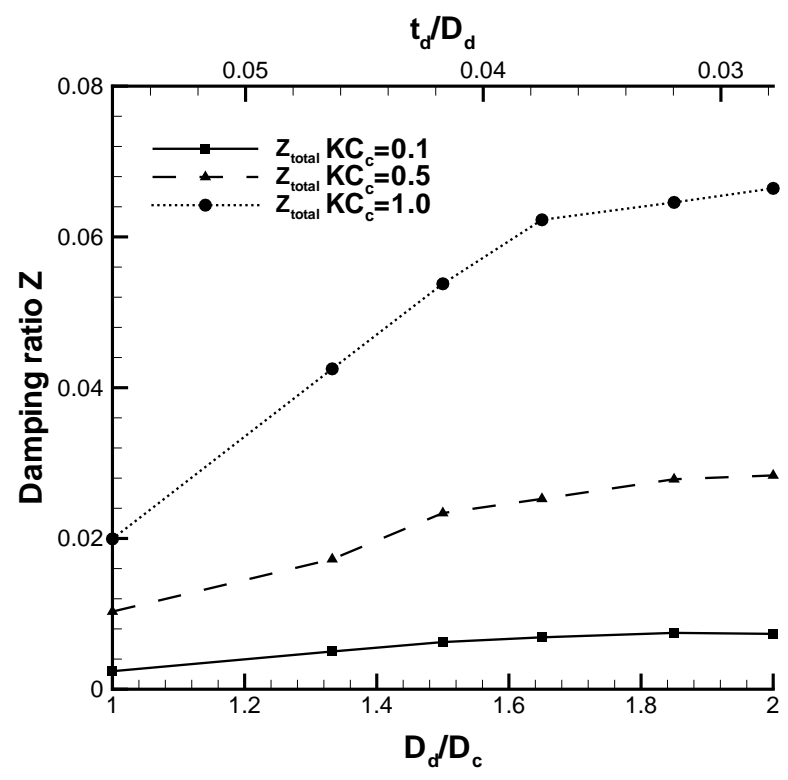

Fig. 10. Damping ratio $Z$ versus $D_{d} / D_{c}$ at different $K C_{c}\left(\beta_{c}=89236\right)$

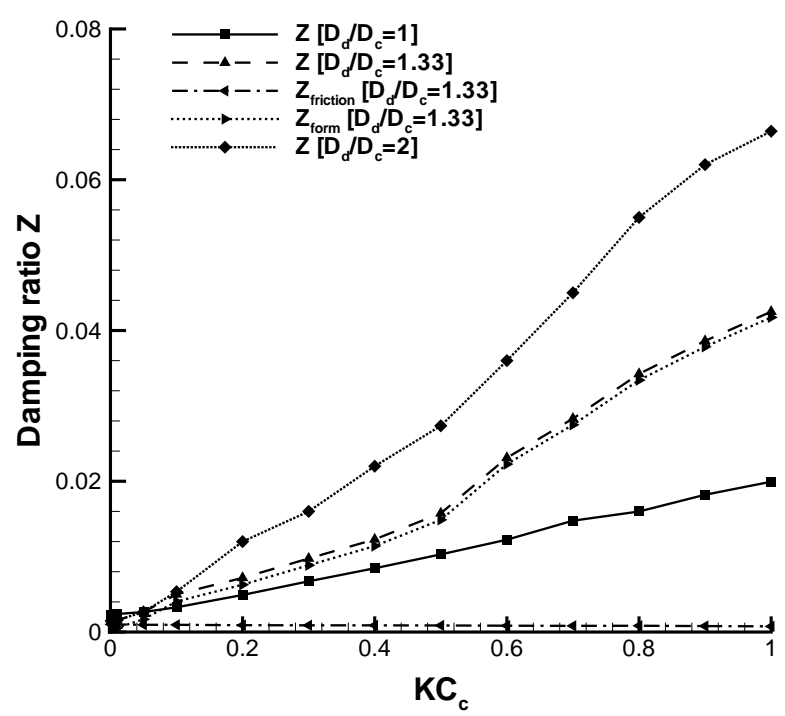

Fig. 11. Damping ratio $Z$ versus $K C_{c}$ at different $D_{d} / D_{c}\left(t_{d} / D_{d}=0.417, \beta_{c}=89236\right)$ 


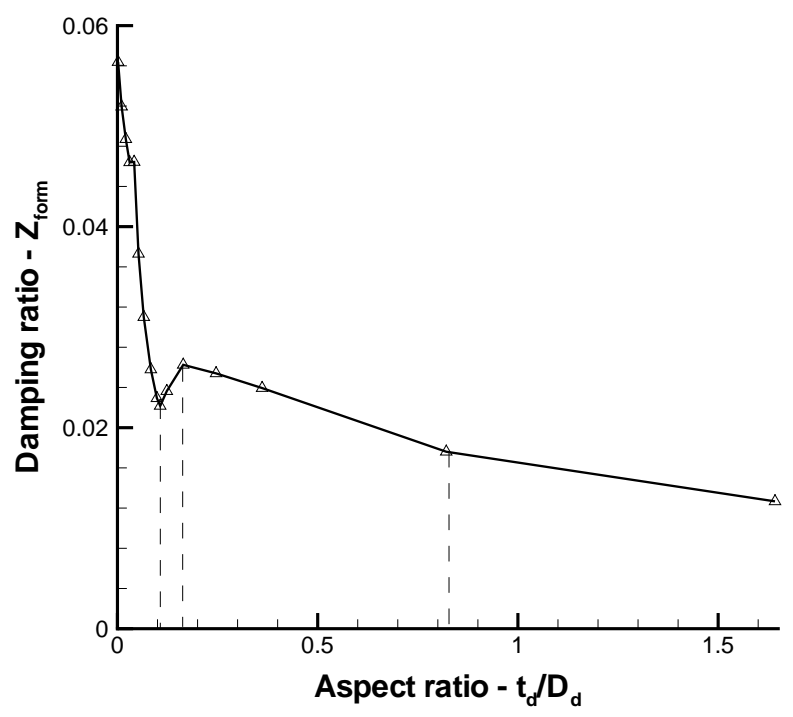

Fig. 12. Effect of aspect ratio $t_{d} / D_{d}$ on heave damping $\left(K C_{c}=1.0\right)$.

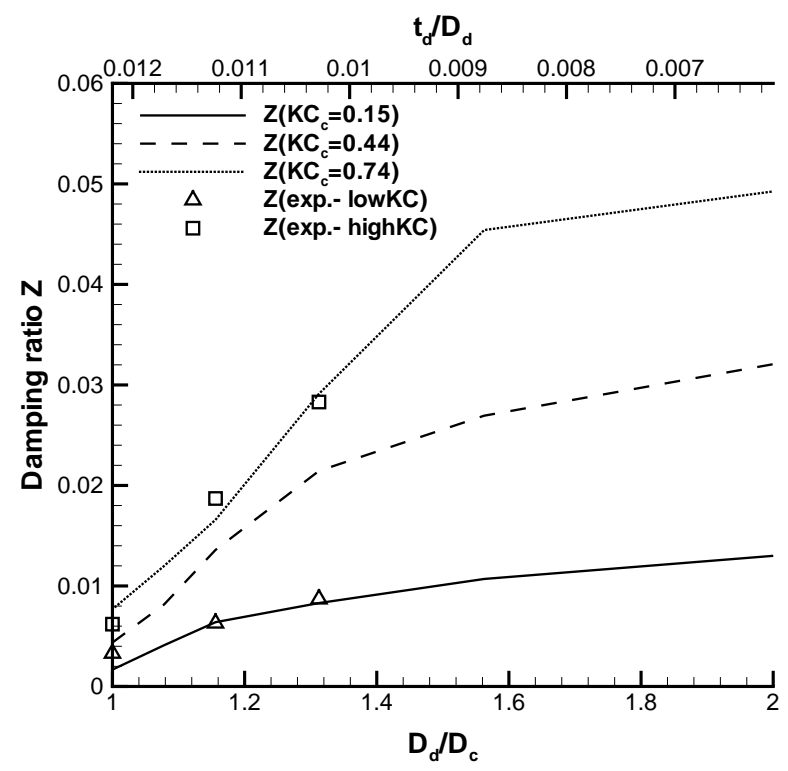

Fig. 13. $Z_{\text {form }}$ versus $D_{d} / D_{c}$ at different $K C_{c}\left(\beta_{c}=4.57 \times 10^{7}\right)$ 